\section{Tetrahedron}

November 2004; 60(45) : 10359-10364

http://dx.doi.org/10.1016/i.tet.2004.07.087

(c) 2004 Elsevier Ltd All rights reserved.
Archimer, archive institutionnelle de l'fremer http://www.ifremer.fr/docelec/

\title{
New access to 1,3-diketones from aldehydes
}

\section{Valérie Fargeas $^{\mathrm{a}^{*}}$, Myriam Baalouch $^{\mathrm{a}}$, Estelle Metay ${ }^{\mathrm{a}}$, Jerôme Baffreau ${ }^{\mathrm{a}}$, Delphine Ménard ${ }^{\mathrm{b}}$, Pascal Gosselin ${ }^{b}$, Jean-Pascal Bergéc ${ }^{c}$, Chantal Barthomeuf ${ }^{\mathrm{d}}$ and Jacques Lebreton ${ }^{\mathrm{a}^{\star \star}}$}

\author{
aLaboratoire de Synthèse Organique, CNRS UMR 6513, Faculté des Sciences et des Techniques, 2 rue de la \\ Houssinière, BP 92208, 44322 Nantes Cedex 3, France \\ ${ }^{\mathrm{b}}$ Laboratoire de Synthèse Organique, CNRS UMR 6511, Faculté des Sciences, Université du Maine, Avenue \\ Olivier Messiaen, F-72085 Le Mans Cedex 9, France \\ 'IFREMER, Département Valorisation des Produits de la Mer, rue de l'Île d'Yeu, B.P. 21105, 44311 Nantes Cedex \\ 3, France \\ UMR-INSERM U-484, Laboratoire de Pharmacognosie et Biotechnologies, Faculté de Pharmacie, Université \\ d'Auvergne, pl. H. Dunant, 63001 Clermont-Fd Cedex, France \\ *: Corresponding author : Tel.: +33-251125403; fax: +33-25112552 (J.L.) fargeas@chimie.univ-nantes.fr \\ ** Tel.: +33-251125403; fax: +33-25112552 (J.L.) lebreton@chimie.univ-nantes.fr
}

\begin{abstract}
A simple and efficient methodology to introduce an 1,3-diketone motif from various aldehyde precursors in three steps with good overall yields is described using $\beta$-ketosulphone 7 as masked equivalent of acetone.

A three-step sequence was studied leading in good yields to 1,3-diketones from aldehydes.
\end{abstract}

Keywords: Aldehydes; Sulfones; Alkylation; 1,3-Diketones 
Pergamon

\title{
New Access to 1,3-Diketones from Aldehydes.
}

\author{
Valérie Fargeas ${ }^{\mathrm{a},{ }^{*}}$, Myriam Baloouch ${ }^{\mathrm{a}}$, Estelle Metay ${ }^{\mathrm{a}}$, Jerôme Baffreau ${ }^{\mathrm{a}}$, Delphine Ménard ${ }^{\mathrm{b}}$, Pascal \\ Gosselin $^{\mathrm{b}}$, Jean-Pascal Bergéc ${ }^{\mathrm{c}}$, Chantal Barthomeuf ${ }^{\mathrm{d}}$ and Jacques Lebreton ${ }^{\mathrm{a}^{* *}}$ \\ a) Laboratoire de Synthèse Organique, CNRS UMR 6513, Faculté des Sciences et des Techniques, 2 rue de la Houssinière, BP 92208 , \\ 44322 Nantes Cedex 3, France. \\ ${ }^{b)}$ Laboratoire de Synthèse Organique, CNRS UMR 6511, Faculté des Sciences, Université du Maine, Avenue Olivier Messiaen, F- \\ 72085 Le Mans Cedex 9 France. \\ c) IFREMER, Département Valorisation des Produits de la Mer, rue de l'île d'Yeu, B.P. 21105, 44311 Nantes Cedex 3, France. \\ d) UMR-INSERM U-484. Laboratoire de Pharmacognosie et Biotechnologies. Faculté de Pharmacie. Université d'Auvergne, pl. H. \\ Dunant, 63001 Clermont-Fd Cedex.
}

\begin{abstract}
A simple and efficient methodology to introduce an 1,3-diketone motif from various aldehyde precursors in three steps with good overall yields is described using $\beta$-ketosulphone 7 as masked equivalent of acetone. (C) 2006 Elsevier Science. All rights reserved
\end{abstract}

Ifremer reported in 1999 the isolation of carotenoid metabolite 1 from the cultured marine micro-algae Skeletonema costatum. ${ }^{1}$ This natural product, for which neither the absolute nor the relative configuration were elucidated, exhibited potent cytotoxicity against a variety of human carcinoma cell lines. ${ }^{2}$ As a part of our interest in the total synthesis of biologically active molecules, especially anti-tumor agents, we drawn our attention towards the synthesis of the metabolite $\mathbf{1}$, and we have established the retrosynthetic plan depicted in Figure 1. Our strategy was centered on introduction of a 1,3-diketone unit from aldehyde 2 at the final stage.

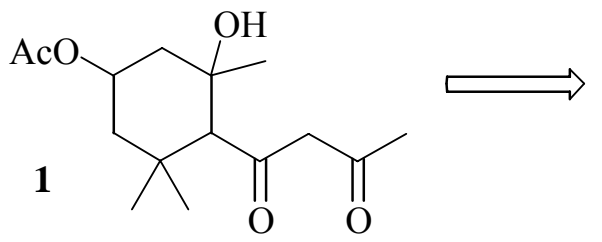<smiles>[2H]C1(C)CC(O)CC(C)(O)C1C=O</smiles>

recently done to minimize side reactions such as $O$ acylation. However, to our knowledge less attention has been devoted to aldehydes. ${ }^{12}$

Figure 1. Retrosynthetic Analysis of marine natural
1,3-Diketones are important building block, and their usefulness in heterocyclic preparations, e.g., pyrazole ${ }^{3}$, isoxazole $^{4}$, triazole $^{5}$ and benzopyran-4-ones ${ }^{6}$ has been largely illustrated. Also, 1,3-diketones are key structural units in many chelating ligand for lanthanide and transition metals. ${ }^{7}$ As a consequence, number of methods have been developed over the years to introduce this moiety which met various degrees of success. One of the most popular ketone precursor, is based on the $\mathrm{C}$-acylation of the corresponding enolates (or silyl enol ethers) with acylating agents, e.g. acid chlorides ${ }^{8}$, acyl cyanides ${ }^{9}$ or 1acylbenzotriazoles ${ }^{10}$, and some improvements ${ }^{11}$ have been approach to introduce the 1,3-diketone motif from the carotenoid metabolite $\mathbf{1 .}$

\footnotetext{
**Corresponding authors. e-mail: fargeas @ chimie.univ-nantes.fr (V. Fargeas); lebreton@chimie.univ-nantes.fr (J. Lebreton).
} 
Also, in order to attach the diketone side chain from 2 to reach our target molecule $\mathbf{1}$, we became interested in developing a good and reliable method compatible with sterically hindered aldehydes. As a model of hindered aldehyde, we chose cyclohexylcarboxaldehyde $\mathbf{3}$ was retained as a bulky model.

Our initial efforts have been to prepare 1,3-diketone from dithiane in an umpolung fashion. Though unprecedented, we tried to condense the lithiated dithiane 4 of the corresponding cyclohexylcarbaldehyde $\mathbf{3}$ with 3chloro-2-(trimethylsiloxy)-1-propene ${ }^{13} \mathbf{5}$ as electrophile acetonylating reagent (see Scheme 1). Unfortunately, all attempts to carry out this reaction in various conditions (bases and additives) were unsuccessful : no reaction take place. By contrast, the addition of more reactive electrophiles such as allybromide ${ }^{14}$ to lithiated dithiane $\mathbf{4}$ led to the formation of the desired product in reasonable yield (60\% yield non-optimized).

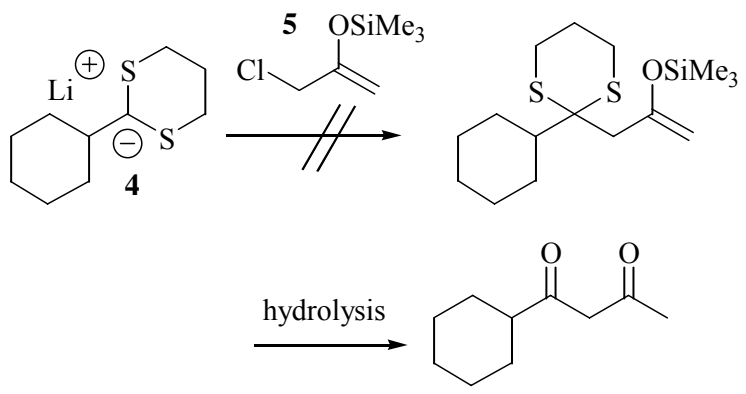

\section{Scheme 1}

Next, it was considered that the monoanion $6^{15}$ of the 1-phenylsulfonylpropanone 7, a masked nucleophilic acetonyl equivalent, could be condensed on the cyclohexylcarbaldehyde $\mathbf{3}$ to afford the corresponding adduct 8 (see Scheme 2). Although, many conditions (NaH/THF, NaH/DMSO, LDA/THF, or DBU/PhH) to generate the stabilized carbanion $\mathbf{6}$ were screened, we always obtained a mixture (vide supra) of starting material, desired adduct $\mathbf{8}$ and unsaturated condensation compound 9. The formation of this latter could be attributed to the acidity of the hydrogen on C-3. Also, quenching conditions were carefully investigated, however the amounts of the byproduct 9 could be only slightly lowered by addition of saturated aqueous ammonium chloride at low temperature.

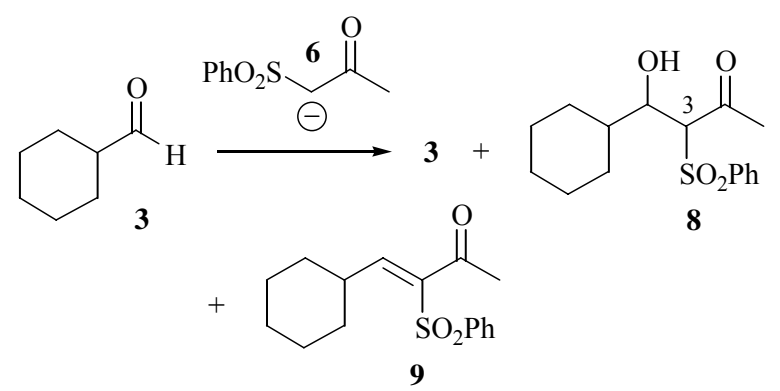

At this point, we turned our attention to an alternative strategy involving the condensation of the dianion $\mathbf{1 0}$ of the 1-phenylsulfonylpropanone 7 on aldehyde 3 (see Scheme 3 ). According to the work of Belletire ${ }^{16}$, the sulfone 7 was treated with 2.5 equimolar amounts of LDA in THF at low temperature to afford the colored dianion $\mathbf{1 0}$ which was then reacted with aldehyde $\mathbf{3}$ to give exclusively after work up the expected aldol $\mathbf{1 1}$ in high yield. In this condensation, no unsaturated compound was detected in the crude mixture (see vide supra). This aldol $\mathbf{1 1}$ was converted by oxidation with the Dess-Martin periodinane ${ }^{17}$ (DMP) into the 1,3-diketone intermediate $\mathbf{1 2}$ in good yield. This method was found to be more efficient compared to PCC and Jones oxidation. Finally, the cleavage of the sulfonyl group of 12 was achieved with sodium-amalgam ${ }^{18}$ to afford the diketone 13 in $77 \%$ yield (45\% overall yield in 3 steps for aldehyde $\mathbf{3}$ ).

Scheme 3: Reagents and conditions: Condensation : (a) 1.6 eq. of 10, THF/HMPA $(7 / 1), 4 \mathrm{~h}$ at $0^{\circ} \mathrm{C}$, then overnight at RT, $86 \%$. Oxidation : (b) 1.0 eq. DMP, $\mathrm{CH}_{2} \mathrm{Cl}_{2}, 6 \mathrm{~h}$, RT,

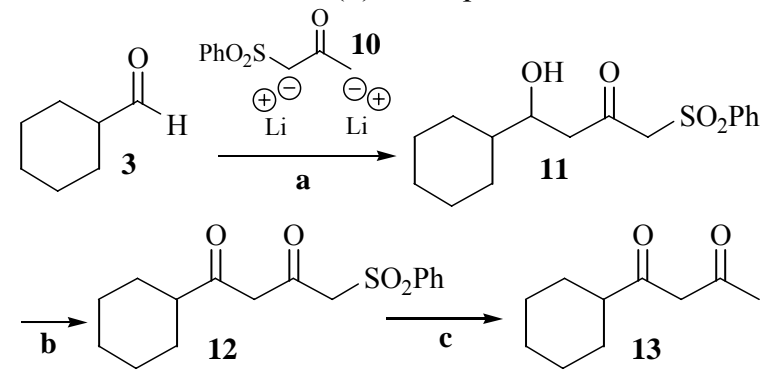

$76 \%$. Desulfonation; (c) $5 \% \mathrm{Na}(\mathrm{Hg}), \mathrm{MeOH},-50^{\circ} \mathrm{C}$, then $2 \mathrm{~h}$ at $-20^{\circ} \mathrm{C}, 77 \%$.

To investigate the potential utility of this methodology, various aldehydes were readily converted to the corresponding diketones as summarized in Table 1. The different intermediates and final products were thus obtained in good to high yields for all steps, expect for the removal of the sulfone group for cinnamaldehyde (entry 2). We were unable to suppress the competitive reduction of the conjugate double on intermediate 17, even using sodium-amalgam with $\mathrm{NaH}_{2} \mathrm{PO}_{4}$ to control $\mathrm{pH}$ of the medium. ${ }^{19}$ All attempts to use samarium(II) iodide to cleave the sulfone group of 17, with HMPA or DMPU as additives failed, leading to complete decomposition of the starting material. ${ }^{20}$ It is especially noteworthy that sodiumamalgam mediated cleavage of sulfones can be applied to substrates containing isolated double bond, such as $\mathbf{2 5}$, in this cases no over reduction occurs.

Scheme 2 
Table 1.

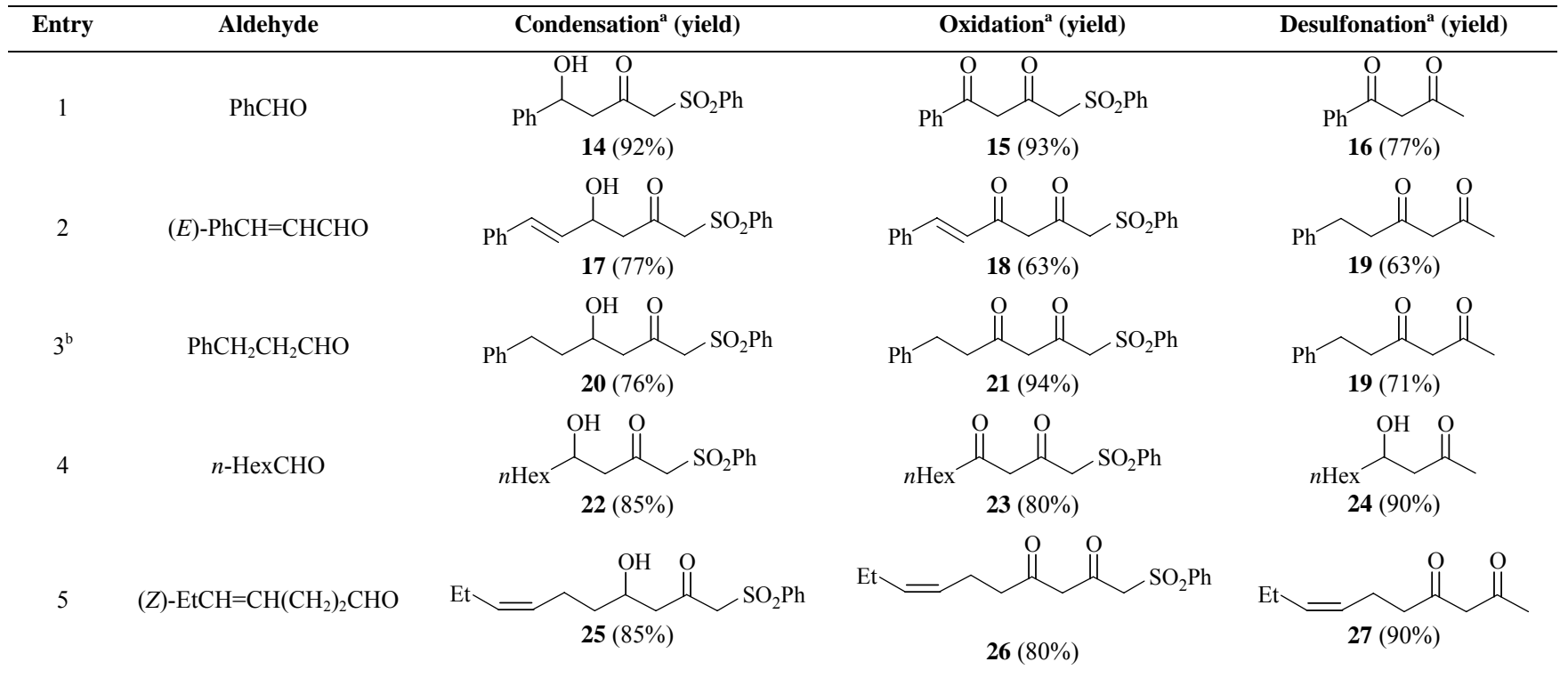

${ }^{\mathrm{a}}$ See conditions Scheme $4 .{ }^{\mathrm{a}}$.

In conclusion, we have developed a simple and efficient methodology to introduce an 1,3-diketone motif from aldehyde precursors in three steps with good overall yields. To evaluate the scope and the efficiency of the present methodology, various aldehydes have been used. We are now extending this methodology to the total synthesis of marine natural carotenoid metabolite $\mathbf{1}$ and the results will be published in due course.

\section{Acknowledgements}

This program is supported by CPER of the Regional Council of the Pays-de-la-Loire and by Ifremer. The authors thank Dr. Patrick Durand (Ifremer) for constant support and encouragements.

\section{Physical data and spectroscopic measurements}

${ }^{1} \mathrm{H}$ NMR spectra were recorded on a Bruker AC 300 instrument at $300 \mathrm{MHz}$. The chemical shifts are expressed (ppm), referenced to residual chloroform (7.26 ppm). Data are reported as follows : $\delta$, chemical shift; multiplicity (recorded as s, singlet; d, doublet; t, triplet; q, quintet and $\mathrm{m}$, multiplet), coupling constants ( $J$ in Hertz, $\mathrm{Hz}$ ), integration and assigment (aromatic, ar). H,H-COSY experiments were routinely carried out to ascertain $\mathrm{H}-\mathrm{H}$ connectivities.

${ }^{13} \mathrm{C}$ NMR spectra were recorded on a Bruker AC 300 instrument at $75 \mathrm{MHz}$. The chemical shifts are expressed (ppm), reported from the central peak of deuterochloroform (76.9 ppm). DEPT (DEPT) experiments were used for evaluating $\mathrm{CH}$ multiplicities. When necessary, ${ }^{13} \mathrm{C}$ spectra were assigned with the aid of HETCOR experiments.

Mass spetra (MS) were obtained on a HP 5889 quadrupolar spectrometer in electronic impact $(70 \mathrm{ev})$ or in chemical ionisation $(500 \mathrm{ev})$ with $\mathrm{NH}_{3}$ gas. HMRS spectra were otained on a JEOL AX500. Mass spectral data are reported as $\mathrm{m} / \mathrm{z}$.

IR spectra were recorded neat in $\mathrm{KBr}$ cells with a Bruker IFS 45 WHR Fourier transform spectrometer. The wave numbers $(v)$ are given in $\mathrm{cm}^{-1}$.

\section{Chromatography}

All reactions were monitored by thin-layer chromatography (TLC) carried out on precoated plate of silica gel 60F 254 (Merk, Art. 5735 alumina sheet).

Flash chromatography was performed on silica gel Merk 60, 230-400 Mesh.

\section{Solvents distillation}

Tetrahydrofuran (THF) was distilled from sodiumbenzophenone. Methanol $(\mathrm{MeOH})$ was distilled from the corresponding magnesium derivative. Dimethylformamide (DMF) was distilled from calcium hydride under reduced presure. 


\section{Usual procedures}

All air and/or water sensitive reactions were carried out under nitrogen atmosphere with dry, freshly distilled solvents using standard syringe/septa techniques.

Yields refer to chromatographically and spectroscopically homogeneous materials, unless otherwise stated.

\section{1-phenylsulfonyl-2-propanone $6:^{16 \mathrm{~b}}$}

To a solution of chloroacetone $(9.2 \mathrm{~mL} ; 114.65 \mathrm{mmol})$ in $150 \mathrm{~mL}$ of freshly distilled DMF was added at RT sodium sulfinate $(18.8 \mathrm{~g} ; 114.65 \mathrm{mmol} ; 1$ eq.). After stirring $24 \mathrm{~h}$ at RT, the reaction mixture was diluted with $20 \mathrm{~mL}^{\text {of }} \mathrm{Et}_{2} \mathrm{O}$ and $20 \mathrm{~mL}$ of water. The aqueous phase was extracted with $3 * 15 \mathrm{~mL} \mathrm{Et}_{2} \mathrm{O}$ before washing with an aqueous $\mathrm{NaCl}$ solution and drying on $\mathrm{MgSO}_{4}$. After evaporation of the solvent, the crude residue was purified on silica gel (eluant : Petroleum ether / ethyl acetate : 7 / 3) to furnish $20 \mathrm{~g}$ of the desired product as pale yellow crystals (Yield $=88 \%$ ). $\mathbf{R M N}{ }^{1} \mathbf{H}\left(\mathbf{C D C l}_{3}\right): \delta=2.24\left(\mathrm{~s}, 3 \mathrm{H}, \mathrm{H}_{3}\right) ; 4.09\left(\mathrm{~s}, 2 \mathrm{H}, \mathrm{H}_{1}\right)$; 7.54-7.67 (m, 3H, $\left.\mathrm{H}_{\mathrm{ar}}\right)$; 7.80-7.94 (m, $\left.2 \mathrm{H}, \mathrm{H}_{\mathrm{ar}}\right) . \mathbf{R M N}{ }^{13} \mathbf{C}$ $\left(\mathbf{C D C l}_{3}\right): \delta=32.4\left(\mathrm{C}_{3}\right) ; 68.7\left(\mathrm{C}_{1}\right) ; 129.1\left(\mathrm{C}_{6}\right.$ and $\left.\mathrm{C}_{6}\right)$; $130.3\left(\mathrm{C}_{5}\right.$ et $\left.\mathrm{C}_{5}\right) ; 135.3\left(\mathrm{C}_{7}\right) ; 139.5\left(\mathrm{C}_{4}\right) ; 197.0\left(\mathrm{C}_{2}\right)$. SM $\left(\mathrm{CI} / \mathrm{NH}_{3}\right): \mathrm{M}+1=199$. IR $: \mathrm{v}=1725 ; 1322 ; 1151$.

\section{A. General procedure for aldolisation :}

To a solution of diisopropylamine $(8.8 \mathrm{~mL} ; 62.5 \mathrm{mmol}$; 2.5 eq.) in $13 \mathrm{~mL}$ of anhydrous $\mathrm{THF}$ at $0^{\circ} \mathrm{C}$ was added dropwise a solution of $n$-butyllithium $1.6 \mathrm{M}$ in hexanes (39 $\mathrm{mL} ; 62.5 \mathrm{mmol} ; 2.5$ eq.) and the solution was stirred during $45 \mathrm{mn}$ before cooling at $-78^{\circ} \mathrm{C}$. At this temperature, $6 \mathrm{~mL}$ of HMPA were added followed by a solution of 1 phenylsulfonyl-2-propanone $(5 \mathrm{~g} ; 25 \mathrm{mmol})$ in $30 \mathrm{~mL}$ of THF. After $4 \mathrm{~h}$ at $0^{\circ} \mathrm{C}$, aldehyde $(32.5 \mathrm{mmol} ; 1.3$ eq.) was added to the resulting orange heterogenous solution and the reaction mixture was allowed to warm to RT overnight. Hydrolysis was achieved at $0^{\circ} \mathrm{C}$ with a saturated aqueous solution of $\mathrm{NH}_{4} \mathrm{Cl}(30 \mathrm{~mL})$, subsequent treatments $\left(\mathrm{Et}_{2} \mathrm{O}\right.$ extraction, drying on $\mathrm{MgSO}_{4}$, concentration and flash chromatography) afforded the desired compound.

4-cyclohexyl-4-hydroxy-1-phenylsulfonyl-2-butanone 11 : (Eluant : $\left.\mathrm{CH}_{2} \mathrm{Cl}_{2}\right) 7 \mathrm{~g}$ of $\mathbf{1 1}$ were obtained as a yellow oil $($ Yield $=86 \%)$. RMN ${ }^{1} \mathbf{H}\left(\mathbf{C D C l}_{3}\right): \delta=1.00-1.84(\mathrm{~m}$, $\left.11 \mathrm{H}, \mathrm{H}_{\text {cyclohexyl }}\right) ; 2.86\left(\mathrm{~d}, J=7,3 \mathrm{~Hz}, 2 \mathrm{H}, \mathrm{H}_{3}\right) ; 2,90(\mathrm{~s}, 1 \mathrm{H}$, $\mathrm{OH}) ; 3.77-3.86\left(\mathrm{~m}, 1 \mathrm{H}, \mathrm{H}_{4}\right) ; 4.22\left(\mathrm{~s}, 2 \mathrm{H}, \mathrm{H}_{1}\right) ; 7.26-7.91$ $\left(\mathrm{m}, 5 \mathrm{H}, \mathrm{H}_{a r}\right) . \mathbf{R M N}{ }^{13} \mathbf{C}\left(\mathbf{C D C l}_{3}\right): \delta=26.0 ; 26.1 ; 26.3$; $28.1 ; 28.8\left(\mathrm{C}_{\text {cyclohexyl }}\right) ; 43.2\left(\mathrm{C}_{\text {cyclohexyl }}\right) ; 48.6\left(\mathrm{C}_{3}\right) ; 67.5$ $\left(\mathrm{C}_{1}\right) ; 71.7\left(\mathrm{C}_{4}\right) ; 128.3\left(2 \mathrm{C}, \mathrm{C}_{\mathrm{ar}}\right) ; 129.4\left(2 \mathrm{C}, \mathrm{C}_{\mathrm{ar}}\right)$; $134.4\left(\mathrm{C}_{\mathrm{ar}}\right) ; 138.7\left(\mathrm{C}_{\mathrm{ar}}\right) ; 199.3\left(\mathrm{C}_{2}\right)$. SM (CI / NH $\left.\mathbf{N H}_{3}\right): \mathrm{M}+1$ = 311. SM (EI) M/Z (\%): 227 (78) ; 199 (66) ; 183 (15) ; 141 (79) ; 77 (100) ; 55 (65) ; 41 (36). IR : 3520; 1715; 1310,1152 .

4-hydroxy-4-phenyl-1-phenylsulfonyl-2-butanone 14 : (Eluants : Petroleum ether / ethyl acetate : 6/4) $7 \mathrm{~g}$ of $\mathbf{1 4}$ were obtained as a yellow oil (Yield $=92 \%$ ). RMN ${ }^{\mathbf{1}} \mathbf{H}$ $\left(\mathbf{C D C l}_{3}\right): \delta=2.96$ and 3.08 (part $\mathrm{AB}$ of an $\mathrm{ABX}$ system, $\left.2 \mathrm{H}, J=17.2 ; 9.2 ; 3.4 \mathrm{~Hz}, \mathrm{H}_{3}\right) ; 4.18\left(\mathrm{~s}, 2 \mathrm{H}, \mathrm{H}_{1}\right) ; 5.07(\mathrm{~m}$, part $\mathrm{X}$ of the $\mathrm{ABX}$ system, $\left.1 \mathrm{H}, \mathrm{H}_{4}\right) ; 7.30-7.80(\mathrm{~m}, 10 \mathrm{H}$, $\left.\mathrm{H}_{\mathrm{ar}}\right)$. RMN ${ }^{13} \mathbf{C}\left(\mathbf{C D C l}_{3}\right): \delta=52.7\left(\mathrm{C}_{3}\right) ; 66.8\left(\mathrm{C}_{1}\right) ; 69.5$ $\left(\mathrm{C}_{4}\right) ; 125.7 ; 127.72 ; 128.2 ; 128.3 ; 129.8 ; 134.2 ; 138.5$; $142,7\left(\mathrm{C}_{\mathrm{ar}}\right) ; 197.7\left(\mathrm{C}_{2}\right)$. MS $\left(\mathrm{CI} / \mathrm{NH}_{3}\right): \mathrm{M}+18=332 ; \mathrm{M}+$ $1=305$. MS (EI) M/Z (\%) : $163(5) ; 141(18) ; 105$ (44) ; 77 (100). IR : $v=3521 ; 1716 ; 1310 ; 1152$.

(5E)-4-hydroxy-6-phenyl-1-phenylsulfonyl-hex-5-en-2one 17 : (Eluants : Petroleum ether / ethyl acetate : 5 / 5 ) $6.3 \mathrm{~g}$ of 17 were obtained as a yellow oil (Yield $=77 \%$ ). RMN ${ }^{1} \mathbf{H} \delta(\mathrm{ppm}): 4.23\left(\mathrm{~s}, 2 \mathrm{H}, \mathrm{H}_{1}\right) ; 3.05\left(\mathrm{~m}, 2 \mathrm{H}, \mathrm{H}_{3}\right)$; 4.75 (qu., $\left.1 \mathrm{H}, \mathrm{H}_{4}\right) ; 6.20\left(\mathrm{dd}, 1 \mathrm{H}, \mathrm{H}_{5}, J=6.2 ; 16 \mathrm{~Hz}\right) ; 6.64$ $\left(\mathrm{d}, 1 \mathrm{H}, \mathrm{H}_{6}, J=16 \mathrm{~Hz}\right) . \mathbf{R M N}{ }^{13} \mathbf{C} \delta(\mathrm{ppm}): 50.9\left(\mathrm{C}_{3}\right) ; 67.5$ $\left(\mathrm{C}_{1}\right) ; 68.4\left(\mathrm{C}_{4}\right) ; 126.6\left(\mathrm{C}_{\mathrm{ar}}\right) ; 134.4\left(\mathrm{C}_{\mathrm{ar}}\right) ; 136.2\left(\mathrm{C}_{7}\right)$; $138.5\left(\mathrm{C}_{\mathrm{ar}}\right) ; 197.8\left(\mathrm{C}_{2}\right)$. MS $\left(\mathrm{CI} / \mathrm{NH}_{3}\right): \mathrm{M}-\left(\mathrm{H}_{2} \mathrm{O}\right)+18=$ 330. MS (EI) M/Z (\%) : $312(5) ; 171$ (93) ; 157 (35); 128 (87) ; 105 (100) ; 77 (48) ; 51 (30) ; 18 (25). IR : 3515 ; $3060 ; 3026 ; 2927 ; 1721 ; 1447 ; 1320 ; 1151 ; 969$; 744 .

\section{4-hydroxy-6-phenyl-1-phenylsulfonyl-2-hexanone 20 :} (Eluants : Petroleum ether / ethyl acetate : 6/4) $6.3 \mathrm{~g}$ of 20 were obtained as a yellow oil (Yield $=76 \%$ ). $\mathbf{R M N}{ }^{\mathbf{1}} \mathbf{H} \delta$ (ppm) : $1.75\left(\mathrm{~m}, 2 \mathrm{H}, \mathrm{H}_{5}\right) ; 2.66\left(\mathrm{~m}, 2 \mathrm{H}, \mathrm{H}_{6}\right) ; 2.82(\mathrm{~d}, 2 \mathrm{H}$, $\left.\mathrm{H}_{3}, J=5.8 \mathrm{~Hz}\right) ; 4.10\left(\mathrm{~m}, 1 \mathrm{H}, \mathrm{H}_{4}\right) ; 4.28\left(\mathrm{~s}, 2 \mathrm{H}, \mathrm{H}_{1}\right) ; 7.18-$ $7.88\left(\mathrm{~m}, 10 \mathrm{H}, \mathrm{H}_{\mathrm{ar}}\right) . \mathbf{R M N}{ }^{13} \mathbf{C} \delta(\mathrm{ppm}): 31.6\left(\mathrm{C}_{6}\right) ; 38.2$ $\left(\mathrm{C}_{5}\right)$; $51.1\left(\mathrm{C}_{3}\right) ; 66.7\left(\mathrm{C}_{4}\right)$; $66.9\left(\mathrm{C}_{1}\right) ; 125.9$; 128.2 ; $128.4 ; 129.3 ; 134.3 ; 138.7 ; 141.6\left(\mathrm{C}_{\mathrm{ar}}\right) ; 198.6\left(\mathrm{C}_{2}\right)$. MS $\left(\mathrm{CI} / \mathrm{NH}_{3}\right): \mathrm{M}+18=350$. MS (EI) M/Z (\%) : $172(35) ; 141$ (21) ; 91 (100) ; 77 (64). IR : 3526 ; 3026 ; 2927 ; 1716 ; $1602 ; 1584 ; 1447 ; 1309 ; 1151 ; 1083 ; 742$.

4-hydroxy-1-phenylsulfonyl-2-decanone 22 : (Eluants : Petroleum ether / ethyl acetate : $6 / 4$ ) $6.6 \mathrm{~g}$ of 22 were obtained as a yellow oil (Yield $=85 \%)$. $\mathbf{R M N}{ }^{1} \mathbf{H} \delta(\mathrm{ppm})$ : $0.87\left(\mathrm{~m}, 3 \mathrm{H}, \mathrm{H}_{10}\right) ; 1.26\left(\mathrm{~m}, 10 \mathrm{H}, \mathrm{H}_{5}, \mathrm{H}_{6}, \mathrm{H}_{7}, \mathrm{H}_{8}, \mathrm{H}_{9}\right) ; 2.81$ $\left(\mathrm{m}, 2 \mathrm{H}, \mathrm{H}_{3}\right) ; 4.03\left(\mathrm{~m}, 1 \mathrm{H}, \mathrm{H}_{4}\right) ; 4.21\left(\mathrm{~s}, 2 \mathrm{H}, \mathrm{H}_{1}\right) ; 7.52-7.90$ $\left(\mathrm{m}, 5 \mathrm{H}, \mathrm{H}_{\mathrm{ar}}\right) . \mathbf{R M N}{ }^{13} \mathrm{C} \delta(\mathrm{ppm}): 14.3\left(\mathrm{C}_{10}\right) ; 22.8\left(\mathrm{C}_{9}\right)$; $25.6\left(\mathrm{C}_{5}\right) ; 29.5\left(\mathrm{C}_{7}\right) ; 32.0\left(\mathrm{C}_{6}\right) ; 36.9\left(\mathrm{C}_{8}\right) ; 51.4\left(\mathrm{C}_{3}\right) ; 67.7$ $\left(\mathrm{C}_{4}\right) ; 67.9\left(\mathrm{C}_{1}\right) ; 128.6\left(2 \mathrm{C} \mathrm{C}_{\mathrm{ar}}\right) ; 129.7\left(2 \mathrm{C} \mathrm{C}_{\mathrm{ar}}\right) ; 134.7$ $\left(\mathrm{C}_{\mathrm{ar}}\right) ; 138.9\left(\mathrm{C}_{\mathrm{ar}}\right) ; 199.2\left(\mathrm{C}_{2}\right)$. MS $\left(\mathrm{CI} / \mathrm{NH}_{3}\right): \mathrm{M}+18=330$. MS (EI) M/Z (\%) : 227 (32); 199 (41) ; 141 (61) ; 77 (100) ; 43 (99). IR : $3521 ; 3063 ; 2925 ; 1716 ; 1585 ; 1494$; $1447 ; 1310 ; 1152 ; 1083 ; 743$.

(7Z)-4-hydroxy-1-phenylsulfonyl-7-decen-2-one 25 : (Eluants : Petroleum ether / ethyl acetate : $6 / 4$ ) $6.6 \mathrm{~g}$ of 25 were obtained as a yellow oil (Yield $=85 \%)$. RMN ${ }^{1} \mathbf{H} \delta$ (ppm) : $0.99\left(\mathrm{~m}, 3 \mathrm{H}, \mathrm{H}_{10}\right) ; 1.52\left(\mathrm{~m}, 2 \mathrm{H}, \mathrm{H}_{6}\right) ; 2.10(\mathrm{~m}, 4 \mathrm{H}$, $\left.\mathrm{H}_{6}, \mathrm{H}_{9}\right) ; 2.84\left(\mathrm{~m}, 2 \mathrm{H}, \mathrm{H}_{3}\right) ; 4.06\left(\mathrm{~m}, 1 \mathrm{H}, \mathrm{H}_{4}\right) ; 4.22(\mathrm{~s}, 2 \mathrm{H}$, $\left.\mathrm{H}_{1}\right)$; $4.06\left(\mathrm{~m}, 2 \mathrm{H}, \mathrm{H}_{7}, \mathrm{H}_{8}\right)$; 7.55-7.92 (m, 5H, $\left.\mathrm{H}_{\mathrm{ar}}\right)$. RMN ${ }^{13} \mathbf{C} \delta(\mathrm{ppm}): 14.3\left(\mathrm{C}_{10}\right) ; 20.5\left(\mathrm{C}_{11}\right) ; 23.1\left(\mathrm{C}_{6}\right) ; 36.4\left(\mathrm{C}_{5}\right)$; $51.1\left(\mathrm{C}_{3}\right) ; 67.2\left(\mathrm{C}_{4}\right) ; 67.5\left(\mathrm{C}_{1}\right) ; 127.8\left(\mathrm{C}_{7}\right.$ or $\left.\mathrm{C}_{8}\right) ; 128.3$ $\left(\mathrm{C}_{\mathrm{ar}}\right) ; 129.4\left(\mathrm{C}_{\mathrm{ar}}\right) ; 132.7\left(\mathrm{C}_{7}\right.$ or $\left.\mathrm{C}_{8}\right) ; 134.4\left(\mathrm{C}_{\mathrm{ar}}\right)$, 138.6 $\left(\mathrm{C}_{\mathrm{ar}}\right) ; 198.7\left(\mathrm{C}_{2}\right)$. MS $\left(\mathrm{CI} / \mathrm{NH}_{3}\right): \mathrm{M}-\left(\mathrm{H}_{2} \mathrm{O}\right)+18=310$. MS (EI) M/Z (\%) : 224 (24) ; 141 (12) ; 83 (100) ; 77 (55) ; 69 (36) ; 55 (31) ; 41 (71) . IR : $3518 ; 3061 ; 1715 ; 1483$; $1310 ; 1152 ; 744$. 


\section{B. General procedure for oxydation :}

To a solution of Dess-Martin periodinane $(0.41 \mathrm{~g} ; 1.0$ mmol ; 1.6 eq.) in $4.4 \mathrm{~mL}$ of $\mathrm{CH}_{2} \mathrm{Cl}_{2}$ was added at $\mathrm{RT}$ a solution of compound to oxidize $(0.6 \mathrm{mmol})$ in $3 \mathrm{~mL}$ of $\mathrm{CH}_{2} \mathrm{Cl}_{2}$. The reaction mixture was stirred $6 \mathrm{~h}$ at $\mathrm{RT}$ then diluted with $15 \mathrm{~mL}$ of $\mathrm{Et}_{2} \mathrm{O}$. $10 \mathrm{~mL}$ of a $10 \%$ aqueous solution of $\mathrm{Na}_{2} \mathrm{~S}_{2} \mathrm{O}_{3}$. and $10 \mathrm{~mL}$ of a $10 \%$ aqueous solution of $\mathrm{NaHCO}_{3}$ were successively added. Subsequent extraction with $\mathrm{Et}_{2} \mathrm{O}(3 * 10 \mathrm{~mL})$ afforded an organic phase which was submitted to usual treatments (drying on $\mathrm{MgSO}_{4}$, concentration and a short flash chromatography) affording the desired compound.

1-cyclohexyl-4-phenylsulfonyl-1,3-butadione 12 : (Eluant : $\left.\mathrm{CH}_{2} \mathrm{Cl}_{2}\right) 150 \mathrm{mg}$ of $\mathbf{1 2}$ were obtained as a yellow oil (Yield $=76 \%)$. RMN ${ }^{1} \mathbf{H}\left(\mathbf{C D C l}_{3}\right) \delta(\mathrm{ppm}): 1.25-1.80$ $\left(\mathrm{m}, 11 \mathrm{H}, \mathrm{H}_{\text {cyclohexyl }}\right) ; 4.02\left(\mathrm{~s}, 2 \mathrm{H}, \mathrm{H}_{3}\right) ; 5.67\left(\mathrm{~s}, 2 \mathrm{H}, \mathrm{H}_{1}\right)$; 7.44-7.59 (m, 3H, $\left.\mathrm{H}_{\mathrm{ar}}\right)$; 7.88-7.98 (m, 2H, $\left.\mathrm{H}_{\mathrm{ar}}\right) . \mathbf{R M N}{ }^{13} \mathbf{C}$ $\left(\mathbf{C D C l}_{3}\right) \delta(\mathrm{ppm}): 25.7 ; 26.1 ; 28.1 ; 28.2 ; 29.4(5 \mathrm{C}$, $\left.\mathrm{C}_{\text {cyclohexyl }}\right) ; 48.6\left(\mathrm{C}_{\text {cyclohexyl }}\right) ; 64.8\left(\mathrm{C}_{1}\right) ; 51.7\left(\mathrm{C}_{3}\right) ; 128.5$ $\left(2 \mathrm{C}, \mathrm{C}_{\mathrm{ar}}\right) ; 129.4\left(2 \mathrm{C}, \mathrm{C}_{\mathrm{ar}}\right) ; 134.3\left(\mathrm{C}_{\mathrm{ar}}\right) ; 138.7\left(\mathrm{C}_{\mathrm{ar}}\right) ; 210.5$ $\left(\mathrm{C}_{4}\right) ; 215.3\left(\mathrm{C}_{2}\right)$. MS $\left(\mathrm{CI} / \mathrm{NH}_{3}\right): \mathrm{M}+1=309$. SM $(\mathrm{EI}) \mathrm{M} / \mathrm{Z}$ $(\%): 308(3) ; 225(100) ; 183(97) ; 141(100) ; 111(23)$; 77 (56) ; 55 (46). IR : $2995 ; 2935 ; 1602 ; 1322 ; 1151$.

\section{1-phenyl-4-phenylsulfonyl-1,3-butanedione}

15 :

(Eluants : Petroleum ether / ethyl acetate : $6 / 4$ ) $0.17 \mathrm{~g}$ of 15 were obtained as a yellow solid (Yield $=93 \%$ ). RMN ${ }^{1} \mathbf{H} \delta(\mathrm{ppm}): 4.17\left(\mathrm{~s}, 2 \mathrm{H}, \mathrm{H}_{1}\right) ; 6.38\left(\mathrm{~s}, 1 \mathrm{H}, \mathrm{H}_{3}\right) ; 7.40-7.80$ $\left(\mathrm{m}, 10 \mathrm{H}, \mathrm{H}_{\mathrm{ar}}\right) . \mathbf{R M N}{ }^{13} \mathbf{C} \delta(\mathrm{ppm}): 31.2\left(\mathrm{C}_{1}\right) ; 65.4\left(\mathrm{C}_{3}\right)$; $127.7 ; 128.5 ; 129.1 ; 129.6 ; 130.4 ; 133.5 ; 134.6 ; 138.8$ $\left(\mathrm{C}_{\mathrm{ar}}\right) ; 184.7\left(\mathrm{C}_{4}\right) ; 208.2\left(\mathrm{C}_{2}\right)$. MS $\left(\mathrm{CI} / \mathrm{NH}_{3}\right): \mathrm{M}+18=320$; $\mathrm{M}+1=303$. MS (EI) M/Z (\%) : 160 (18); 147 (37) ; 118 (35) ; 105 (100) ; 77 (59). IR : $2995 ; 2936 ; 1603 ; 1573$; $1451 ; 1308 ; 1159 ; 1085 ; 694$.

(5E)-6-phenyl-1-phenylsulfonyl-hexene-2,4-dione 18 : (Eluants : Petroleum ether / ethyl acetate : 3 / 7 ) $0.63 \mathrm{~g}$ of 17 were obtained as a red oil (Yield $=63 \%$ ). $\mathbf{R M N}{ }^{\mathbf{1}} \mathbf{H} \delta$ (ppm): $4.13\left(\mathrm{~s}, 2 \mathrm{H}, \mathrm{H}_{1}\right) ; 5.92\left(\mathrm{~s}, 1 \mathrm{H}, \mathrm{H}_{3}\right) ; 6.51\left(\mathrm{~d}, 1 \mathrm{H}, \mathrm{H}_{6}\right.$, $J=15.8 \mathrm{~Hz}) ; 7.29-7.68\left(\mathrm{~m}, 11 \mathrm{H}, \mathrm{H}_{\mathrm{ar}}\right.$ and $\left.\mathrm{H}_{5}\right) . \mathbf{R M N}{ }^{13} \mathbf{C} \delta$ (ppm): $65.9\left(\mathrm{C}_{1}\right) ; 102.4\left(\mathrm{C}_{6}\right) ; 121.8-141.9\left(14 \mathrm{C}, 12 \mathrm{C}_{\mathrm{ar}}, \mathrm{C}_{5}\right.$, $\left.\mathrm{C}_{3}\right) ; 178.5\left(\mathrm{C}_{4}\right) ; 185.8\left(\mathrm{C}_{2}\right)$. MS $\left(\mathrm{CI} / \mathrm{NH}_{3}\right): \mathrm{M}+18=346$; $\mathrm{M}+1=329$. MS (EI) M/Z (\%) : 312 (12) ; 171 (100) ; 157 (33) ; 128 (61) ; 105 (65) ; 77 (35) ; 51 (15). IR : 3439 ; $3060 ; 3028$; $2925 ; 1633$; $1578 ; 1446$; 1313 ; 1307 ; $1156 ; 1084$.

6-phenyl-1-phenylsulfonyl-hexane-2,4-dione 19 : (Eluants : Petroleum ether / ethyl acetate : 6 / 4 ) $0.186 \mathrm{~g}$ of 18 were obtained as a brown oil (Yield $=94 \%$ ). RMN ${ }^{1} \mathbf{H}$ $\delta(\mathrm{ppm}): 2.78\left(\mathrm{~m}, 4 \mathrm{H}, \mathrm{H}_{5}\right.$ and $\left.\mathrm{H}_{6}\right) ; 4.00\left(\mathrm{~s}, 2 \mathrm{H}, \mathrm{H}_{1}\right) ; 5.69$ $\left(\mathrm{s}, 1 \mathrm{H}, \mathrm{H}_{3}\right) ; 7.16-8.00\left(\mathrm{~m}, 10 \mathrm{H}, \mathrm{H}_{\mathrm{ar}}\right) . \mathbf{R M N}{ }^{13} \mathbf{C} \delta(\mathrm{ppm})$ : $31.5\left(\mathrm{C}_{6}\right) ; 40.3\left(\mathrm{C}_{5}\right) ; 64.7\left(\mathrm{C}_{1}\right) ; 102.6\left(\mathrm{C}_{3}\right) ; 126.7 ; 128.6$ ; $128.7 ; 128.9 ; 129.5 ; 134.6 ; 138.8 ; 140.4\left(\mathrm{C}_{\mathrm{ar}}\right) ; 179.4$ $\left(\mathrm{C}_{2}\right) ; 195.1\left(\mathrm{C}_{4}\right)$. MS $\left(\mathrm{CI} / \mathrm{NH}_{3}\right): \mathrm{M}+18=348 ; \mathrm{M}+1=331$. MS (EI) M/Z (\%) : $188(27) ; 141(30) ; 131(54) ; 104(62)$ ; 91 (100) ; 77 (81). IR :3062 ; 3027 ; 2927 ; 1721 ; 1602 ; $1496 ; 1447 ; 1323 ; 1310 ; 1153 ; 1083 ; 700$. 1-phenylsulfonyl-decane-2,4-dione 23 : (Eluants : Petroleum ether / ethyl acetate : $6 / 4$ ) $0.15 \mathrm{~g}$ of 23 were obtained as a yellow oil (Yield $=80 \%) . \mathbf{R M N}{ }^{1} \mathbf{H} \delta(\mathrm{ppm})$ : $0.86\left(\mathrm{~m}, 3 \mathrm{H}, \mathrm{J}=7 \mathrm{~Hz}, \mathrm{H}_{10}\right) ; 1.27$ (m, 8H, H$\left., \mathrm{H}_{7}, \mathrm{H}_{8}, \mathrm{H}_{9}\right)$; $1.55\left(\mathrm{~m}, 2 \mathrm{H}, \mathrm{H}_{5}\right) ; 4.00\left(\mathrm{~s}, 2 \mathrm{H}, \mathrm{H}_{1}\right) ; 5.65\left(\mathrm{~s}, 1 \mathrm{H}, \mathrm{H}_{3}\right) ; 7.49-$ $7.90\left(\mathrm{~m}, 5 \mathrm{H}, \mathrm{H}_{\mathrm{ar}}\right) . \mathbf{R M N}{ }^{13} \mathbf{C} \delta(\mathrm{ppm}): 14.3\left(\mathrm{C}_{10}\right) ; 22.7$ $\left(\mathrm{C}_{9}\right) ; 25.7\left(\mathrm{C}_{5}\right) ; 29.0\left(\mathrm{C}_{7}\right) ; 31.7\left(\mathrm{C}_{6}\right) ; 38.6\left(\mathrm{C}_{8}\right) ; 64.7\left(\mathrm{C}_{1}\right)$ ; $102.2\left(\mathrm{C}_{3}\right) ; 128.7\left(2 \mathrm{C}, \mathrm{C}_{\mathrm{ar}}\right) ; 129.5\left(2 \mathrm{C}, \mathrm{C}_{\mathrm{ar}}\right) ; 134.5\left(\mathrm{C}_{\mathrm{ar}}\right)$; $138.9\left(\mathrm{C}_{\mathrm{ar}}\right) ; 179.9\left(\mathrm{C}_{4}\right) ; 196.2\left(\mathrm{C}_{2}\right)$. MS $\left(\mathrm{CI} / \mathrm{NH}_{3}\right): \mathrm{M}+18$ $=328 ; \mathrm{M}+1=311$. MS $(\mathrm{EI}) \mathrm{M} / \mathrm{Z}(\%): 240(38) ; 225(16)$ ; 183 (49) ; 169 (33) ; 141 (98) ; 125 (29) ; 113 (40) ; 99 (41) ; 85 (75) ; 77 (100) ; 69 (24) ; 55 (36) ; 43 (91). IR : $2995 ; 2936 ; 1602 ; 1445 ; 1317 ; 1308 ; 1159 ; 1085$; 694.

(7Z)-1-phenylsulfonyl-decene-2,4-dione 26 : (Eluants : Petroleum ether / ethyl acetate : $6 / 4$ ) $0.15 \mathrm{~g}$ of $\mathbf{2 6}$ were obtained as a yellow oil (Yield $=80 \%) . \mathbf{R M N}^{1} \mathbf{H} \delta(\mathrm{ppm})$ : $0.93\left(\mathrm{t}, 3 \mathrm{H}, J=7 \mathrm{~Hz}, \mathrm{H}_{10}\right) ; 1.90-2.15\left(\mathrm{~m}, 2 \mathrm{H}, \mathrm{H}_{9}\right)$; 2.19$2.45\left(\mathrm{~m}, 4 \mathrm{H}, \mathrm{H}_{5}\right.$ and $\left.\mathrm{H}_{6}\right) ; 4.02\left(\mathrm{~s}, 2 \mathrm{H}, \mathrm{H}_{1}\right) ; 5.15-5.62(\mathrm{~m}$, $2 \mathrm{H}, \mathrm{H}_{3}$ and $\left.\mathrm{H}_{4}\right) ; 5.70\left(\mathrm{~s}, 1 \mathrm{H}, \mathrm{H}_{3}\right) ; 7.49-7.99\left(\mathrm{~m}, 5 \mathrm{H}, \mathrm{H}_{\mathrm{ar}}\right)$. $\mathbf{R M N}{ }^{13} \mathbf{C} \delta(\mathrm{ppm}): 14.2\left(\mathrm{C}_{10}\right) ; 20.5\left(\mathrm{C}_{9}\right) ; 23.0\left(\mathrm{C}_{5}\right.$ or $\left.\mathrm{C}_{6}\right)$; $38.4\left(\mathrm{C}_{5}\right.$ or $\left.\mathrm{C}_{6}\right) ; 64.5\left(\mathrm{C}_{1}\right) ; 102.2\left(\mathrm{C}_{7}\right.$ and $\left.\mathrm{C}_{8}\right) ; 126.3$, 128.4, 129.4, 134.3, $138.5\left(5 \mathrm{C}, \mathrm{C}_{\mathrm{ar}}\right), 179.8\left(\mathrm{C}_{4}\right) ; 195.1$ $\left(\mathrm{C}_{2}\right)$. MS $\left(\mathrm{CI} / \mathrm{NH}_{3}\right): \mathrm{M}+18=326 ; \mathrm{M}+1=309$. MS (EI) $\mathrm{M} / \mathrm{Z}(\%): 240(56) ; 225(12) ; 199(15) ; 183(29) ; 167$ (26); $141(77) ; 125(31) ; 109(53) ; 77(100) ; 67(48) ; 55$ (46) ; 41 (75). IR : $2957 ; 2930 ; 1615 ; 1506 ; 1446 ; 1320$ ; $1309 ; 1156 ; 1085$.

\section{General procedure for desulfonation :}

Turnings of sodium $(0.46 \mathrm{~g} ; 20 \mathrm{mmol} ; 12.2$ eq. $)$ were added to mercury $(7.3 \mathrm{~g} ; 36.4 \mathrm{mmol} ; 22$ eq. $)$ at RT. $8.3 \mathrm{~mL}$ of dry $\mathrm{MeOH}$ were then added to the corresponding amalgame and the temperature was cooled to $-50^{\circ} \mathrm{C}$. A solution of 1,3-dione (1.7 mmol ; 1 eq.) in $12 \mathrm{~mL}$ of $\mathrm{MeOH}$ was added and the reaction mixture was stirred $2 \mathrm{~h}$ at $-20^{\circ} \mathrm{C}$ before the hydrolysis was achieved with a saturated aquous solution of $\mathrm{NH}_{4} \mathrm{Cl}(40 \mathrm{~mL})$ at $0^{\circ} \mathrm{C}$ After filtration, the aqueous phase was extracted with $3 * 10 \mathrm{~mL} \mathrm{Et}_{2} \mathrm{O}$ before drying on $\mathrm{MgSO}_{4}$. After evaporation of the solvent, the crude residue was purified by a short flash chromatography to afford the desired compound. All compounds were obtained under their enolic form.

1-cyclohexyl-butane-1,3-dione 13: (Eluants: $\mathrm{CH}_{2} \mathrm{Cl}_{2}$ ) $0.22 \mathrm{mg}$ of 13 were obtained as a pale orange oil (Yield = 77 \%). RMN ${ }^{1} \mathbf{H} \quad\left(\mathbf{C D C l}_{3}\right) \delta(\mathrm{ppm})$ : 0.69-1.89 (m, $\left.10 \mathrm{H}, \mathrm{H}_{\text {cyclohexyl }}\right) ; 2.06\left(\mathrm{~s}, 3 \mathrm{H}, \mathrm{H}_{4}\right) ; 2.22\left(\mathrm{~m}, 1 \mathrm{H}, \mathrm{H}_{\text {cyclohexyl }}\right)$; $3.60\left(\mathrm{~s}, 2 \mathrm{H}, \mathrm{H}_{2}\right) ; 5.48\left(\mathrm{~s}, 1 \mathrm{H}, \mathrm{H}_{2}\right) ; 12.06(\mathrm{~s}, 1 \mathrm{H}, \mathrm{OH})$. $\mathbf{R M N}^{13} \mathbf{C} \delta(\mathrm{ppm}): 24.0 ; 25.9 ; 27.8 ; 29.6$ (4C, $\left.\mathrm{C}_{\text {cyclohexyl }}\right)$ ; $28.5\left(\mathrm{C}_{4}\right) ; 46.4\left(\mathrm{C}_{5}\right) ; 98.1\left(\mathrm{C}_{2}\right) ; 192.6\left(\mathrm{C}_{1}\right.$ and $\left.\mathrm{C}_{3}\right)$. MS $\left(\mathrm{CI} / \mathrm{NH}_{3}\right): \mathrm{M}+1=169$. MS (EI) M/Z (\%) : $168(18) ; 113$ (12) ; 85 (100); 55 (24) ; 43 (35). HR MS : 168.1167 $\left(\mathrm{C}_{10} \mathrm{H}_{16} \mathrm{O}_{2}\right.$; calcd 168.1150). IR : $2966 ; 1612 ; 1492$; 1364. 
1-phenyl-butan-1,3-dione 16 : (Eluants : Petroleum ether / ethyl acetate : 7 / 3) $140 \mathrm{mg}$ of $\mathbf{1 6}$ were obtained as a pale yellow oil (Yield $=76 \%)$. RMN ${ }^{1} \mathbf{H} \delta(\mathrm{ppm}): 2.06(\mathrm{~s}, 3 \mathrm{H}$, $\left.\mathrm{H}_{1}\right) ; 5.03\left(\mathrm{~m}, 1 \mathrm{H}, \mathrm{H}_{3}\right) ; 7.15-7.24\left(\mathrm{~m}, 5 \mathrm{H}, \mathrm{H}_{\mathrm{ar}}\right)[10 \%$ of the 1-3 diketone form is detected : $2.18\left(\mathrm{~s}, 3 \mathrm{H}, \mathrm{H}_{1}\right) ; 6.20(\mathrm{~s}$, $\left.\left.2 \mathrm{H}, \mathrm{H}_{3}\right) ; 7.43-7.84\left(\mathrm{~m}, 5 \mathrm{H}, \mathrm{H}_{\mathrm{ar}}\right)^{21}\right] . \mathbf{R M N}{ }^{13} \mathbf{C} \delta(\mathrm{ppm})$ : $24.1\left(\mathrm{C}_{1}\right) ; 47.0\left(\mathrm{C}_{3}\right) ; 68.8\left(\mathrm{C}_{2}\right) ; 75.2\left(\mathrm{C}_{4}\right) ; 125.7\left(2 \mathrm{C}, \mathrm{C}_{\mathrm{ar}}\right)$ ; 127.5 (Car) ; $128.5\left(2 \mathrm{C}, \mathrm{C}_{\mathrm{ar}}\right) ; 144.5\left(\mathrm{C}_{\mathrm{ar}}\right)$. MS $\left(\mathrm{CI} / \mathrm{NH}_{3}\right)$ : $\mathrm{M}+1=163 ; \mathrm{M}+18=180$. MS (EI) M/Z (\%) : 146 (28); 105 (53) ; 77 (67) ; 43 (98). IR : $3436 ; 3062 ; 3030 ; 2925$ ; 1713 ; 1603 ; $1494 ; 1449 ; 1360 ; 1323$; 1158 ; 1083 ; $756 ; 701$.

6-phenyl-hexan-2,4-dione 19 : (Eluants : Petroleum ether / ethyl acetate : 7 / 3) $224 \mathrm{mg}$ of 19 were obtained as a brown oil (Yield = $71 \%)$. RMN ${ }^{1} \mathbf{H} \delta(\mathrm{ppm}): 2.04\left(\mathrm{~s}, 3 \mathrm{H}, \mathrm{H}_{1}\right)$; $2.65\left(\mathrm{~m}, 2 \mathrm{H}, \mathrm{H}_{5}\right) ; 2.92\left(\mathrm{~m}, 2 \mathrm{H}, \mathrm{H}_{6}\right) ; 5.48\left(\mathrm{~s}, 1 \mathrm{H}, \mathrm{H}_{3}\right)$; 7.17-7.29 (m, 5H, $\left.\mathrm{H}_{\mathrm{ar}}\right)$. RMN ${ }^{13} \mathbf{C} \delta(\mathrm{ppm}): 25.1\left(\mathrm{C}_{5}\right) ; 31,8$ $\left(\mathrm{C}_{1}\right) ; 40.3\left(\mathrm{C}_{3}\right) ; 100.3\left(\mathrm{C}_{6}\right) ; 126.5\left(\mathrm{C}_{\mathrm{ar}}\right) ; 128.6\left(2 \mathrm{C}, \mathrm{C}_{\mathrm{ar}}\right)$; $128.8\left(2 \mathrm{C}, \mathrm{C}_{\mathrm{ar}}\right) ; 140.9\left(\mathrm{C}_{\mathrm{ar}}\right) ; 191.3\left(\mathrm{C}_{4}\right) ; 193.5\left(\mathrm{C}_{2}\right) . \mathbf{M S}$ $\left(\mathrm{CI} / \mathrm{NH}_{3}\right): \mathrm{M}+1=191 ; \mathrm{M}+18=208 . \mathbf{S M}(\mathrm{EI}) \mathrm{M} / \mathrm{Z}(\%): 190$ (21) ; 104 (67) ; 91 (100) ; 77 (30) ; 43 (100). HR MS : $190.0996\left(\mathrm{C}_{12} \mathrm{H}_{14} \mathrm{O}_{2}\right.$; calcd 190.0994) IR : $3027 ; 2928$; $1706 ; 1603 ; 1496 ; 1454 ; 1361 ; 1134 ; 785 ; 750 ; 699$.

decane-2,4-dione 24 : (Eluants : Petroleum ether / ethyl acetate : 7 / 3) $265 \mathrm{mg}$ of $\mathbf{2 4}$ were obtained as a yellow oil $($ Yield $=90 \%) . \mathbf{R M N}{ }^{1} \mathbf{H} \delta(\mathrm{ppm}): 0.86\left(\mathrm{~m}, 3 \mathrm{H}, \mathrm{H}_{10}\right)$; $1.26\left(\mathrm{~m}, 16 \mathrm{H}, \mathrm{H}_{6}-\mathrm{H}_{9}\right) ; 2.02\left(\mathrm{~m}, 3 \mathrm{H}, \mathrm{H}_{1}\right) ; 2.22\left(\mathrm{~m}, 2 \mathrm{H}, \mathrm{H}_{5}\right)$ ; $5.47\left(\mathrm{~s}, 1 \mathrm{H}, \mathrm{H}_{3}\right) . \mathbf{R M N}{ }^{13} \mathbf{C} \delta(\mathrm{ppm}): 14.2 ; 22.7 ; 25.2$; $25.9 ; 29.1\left(5 \mathrm{C}, \mathrm{C}_{\mathrm{alk}}\right) ; 31.8\left(\mathrm{C}_{1}\right) ; 38.5\left(\mathrm{C}_{\mathrm{alk}}\right) ; 99.9\left(\mathrm{C}_{3}\right)$; $191.7\left(\mathrm{C}_{4}\right) ; 194.5\left(\mathrm{C}_{2}\right)$. MS $\left(\mathrm{CI} / \mathrm{NH}_{3}\right): \mathrm{M}+1=171$; $\mathrm{M}+18=188$. MS (EI) M/Z (\%) : $170(2) ; 113(18) ; 100$ (55) ; 85 (100) ; 72 (16) ; 43 (100). HR MS : 170.1304 $\left(\mathrm{C}_{10} \mathrm{H}_{18} \mathrm{O}_{2}\right.$; calcd 170.130). IR : $2956 ; 2929 ; 2858 ; 1613$; $1460 ; 1364$.

(7Z)-decene-2,4-dione 27 : (Eluants : Petroleum ether / ethyl acetate: 7 / 3) $265 \mathrm{mg}$ of 27 were obtained as a yellow oil $($ Yield $=90 \%)$. RMN ${ }^{1} \mathbf{H} \delta(\mathrm{ppm}): 0.88(\mathrm{t}, 3 \mathrm{H}$, $\left.J=7 \mathrm{~Hz}, \mathrm{H}_{10}\right) ; 1.95\left(\mathrm{~s}, 3 \mathrm{H}, \mathrm{H}_{1}\right) ; 1.95\left(\mathrm{~m}, 2 \mathrm{H}, \mathrm{H}_{9}\right) ; 2.25$ $\left(\mathrm{m}, 4 \mathrm{H}, \mathrm{H}_{5}-\mathrm{H}_{6}\right) ; 5.15-5.41\left(\mathrm{~m}, 2 \mathrm{H}, \mathrm{H}_{7}-\mathrm{H}_{8}\right) ; 5.42(\mathrm{~s}, 1 \mathrm{H}$, $\left.\mathrm{H}_{3}\right) . \mathbf{R M N}{ }^{13} \mathbf{C} \delta(\mathrm{ppm}): 13\left(\mathrm{C}_{10}\right) ; 20\left(\mathrm{C}_{9}\right) ; 22\left(\mathrm{C}_{6}\right) ; 24$ $\left(\mathrm{C}_{1}\right) ; 37\left(\mathrm{C}_{5}\right) ; 99\left(\mathrm{C}_{3}\right) ; 126$ and $132\left(\mathrm{C}_{7}\right.$ and $\left.\mathrm{C}_{8}\right) ; 190$ and $192\left(\mathrm{C}_{2}\right.$ and $\left.\mathrm{C}_{4}\right)$. MS $\left(\mathrm{CI} / \mathrm{NH}_{3}\right): \mathrm{M}+1=169 ; \mathrm{M}+18=186$. MS (EI) M/Z (\%) : $168(2) ; 110(11) ; 100(30) ; 85$ (100); 67 (27) ; 55 (14) ; 43 (71). HR MS : $168.1154\left(\mathrm{C}_{10} \mathrm{H}_{16} \mathrm{O}_{2}\right.$; calcd 168.1150). IR : $2956 ; 2863 ; 1713 ; 1620 ; 1454$; $1360 ; 1142$.

1. WO 0044718 A1 20000803 or C. A. 133, 134246.

2. Bergé, J. P.; Bourgougnon, N. ; Carbonnelle, D. ; Le Bert, V. ; Tomasoni, C. ; Durand, P.; Roussakis, C. Anticancer Res. 1997, 17, 2115-2120.

3. Nagpal, A.; Unny, R.; Joshi, Y. C. Heterocyclic Commun. 2001, 32, 589-592.

4. Simoni, D.; Invidiata, F. P. ; Rondanin, R. ; Grimaudo, S. ; Cannizzo, G. ; Barbusca, E.; Porretto, F.; D'Alessandro, N.; Tolomeo, M. J. Med. Chem. 1999, 42, 4961-4969.
5. Alekseev, V. V.; Zelinin, K. N.; Yakimovich, S. I. Russ. J. Org. Chem. 1995, 31, 705-727.

6. (a) Ellis, G. P. The Chemistry of Heteroclic Compounds. In Chromanones, and Chromones; Ellis, G. P., Ed.; Interscience: USA, 19; vol. 33, pp 495-555. (b) Raston, C. L. ; Salem, G. J. Chem. Soc., Chem. Commun. 1984, 1702-1703.

7. Garnovskii, A. D.; Kharixov, B. I.; Blanco, L. M.; Garnovskii, D. A.; Burlov, A. S.; Vasilchenko, I. S.; Bondarenko, G. I. J. Coord. Chem. 1999, 46, 365-375.

8. Beck, A. K.; Hoekstra, M. S.; Seebach, D. Tetrahedron Lett. 1977, 18, 1187-1190.

9. Tang, Q.; Sen, S. E. Tetrahedron Lett. 1998, 39, 2249-2252.

10. Katritzky, A. R.; Pastor, A. J. Org. Chem. 2000, 65, 36793682.

11. (a) Le Roux, C. ; Mandrou, S. ; Dubac, J. J. Org. Chem. 1996, 61, 3885-3887 and references cited herein. (b) For recent reference, see: (b) Wiles, C.; Watts, P.; Haswell, S. J; Pombo-Villar, E. Tetrahedron Lett. 2002, 43, 2945-2948. (c) Kel'in, A. V. Curr. Org. Chem. 2003, 7, 1-21.

12. Ballini, R.; Bartoli, G. Synthesis 1993, 965-967.

13. Hosomi, H; Shirahata, A.; Araki, Y.; Sakurai, H. J. Org. Chem. 1981, 46, 4631-4633.

14. For similar examples, see: (a) Page, P. C. B.; McKenzie, M. J.; Buckle, D. R. Tetrahedron 1998, 54, 14581-14596. (b) Jia, Y. X. Li, X.; Wu, B.; Zhao, X. Z.; Tu, Y. Q. Tetrahedron 2002, 58, 1697-1708.

15. Giblin, G. M. P.; Simpkins, N. S. J. Chem. Soc., Chem. Commun. 1987, 207-208. For a good preparation of 1phenylsulfonylpropanone 7 see : Tavares, D. F.; O'Sullivan, W. I.; Hauser, C. R. J. Org. Chem. 1962, 27, 1251-1254.

16. (a) Belletire, J. L. ; Spletzer, E. G. Synthetic Com. 1987, 17, 1701-1707. (b) Wada, E.; Pei, W.; Yasuoka, H. ; Chin, U.; Kanemasa, S. Tetrahedron 1996, 52, 1205-1220.

17. (a) Dess, D. B.; Martin, J. C. J. Org. Chem. 1983, 48, 41554156. (b) Meyer, S. D.; Schreiber, S. L. J. Org. Chem. 1994, 59, 7549-7552.

18. See: Kocienski, P. J.; Lythgoe, B.; Ruston, S. J. Chem. Soc., Perkins Trans. I 1978, 829-834.

19. Trost, B. M.; Arndt, H. C. ; Strege, P. E.; Verhoeven, T. R. Tetrahedron Lett. 1976, 39, 3477-3478.

20. Molander, G. A.; Hahn, G.; J. Org. Chem. 1986, 51, 11351138. For more recent examples, see: Colucci, J.; Lee, D.; Wilson, M.-C. Org. Lett. 2002, 4, 4705-4706.

21. Wiles, C.; Watts, P.; Haswell, S. J.; Pombo-Villar, E. Tetrahedron Lett. 2002, 43, 2945-2948. 